\title{
FUSARIUM SPP.: A RARE CUTANEOUS OPPORTUNISTIC INFECTION IN A WOMAN WITH SYSTEMIC LUPUS ERYTHEMATOSUS (SLE) AND MULTIPLE SKIN LESIONS
}

Victor Junio Pereira Mesquita ${ }^{1, \star}$, Paula Cristina Montina ${ }^{1}$, Jamille Nascimento Carneiro ${ }^{1}$, Regina Alice Fontes Von Kirchenheim ${ }^{1}$ Carlos Eduardo De Carvalho Lins ${ }^{1}$

1.Hospital de Base do Distrito Federal, Brasília (DF), Brazil.

*Corresponding author: victorjunio.ptu@hotmail.com

\section{BACKGROUND}

Patients with SLE appear to have an increased risk for infections due to defects of the immune system. Disease activity is a risk factor for infections, especially when it includes organ involvement such as the kidney. Fungal diseases are common and potentially serious in immunosuppressed patients. Fusarium spp. is an opportunistic fungus. Fusarium infection can be localized, focally invasive, or disseminated and represents a complication of high morbidity and mortality in immunocompromised. We report a case of fusariosis in an immunocompromised patient with refractory lupus nephritis.

\section{CASE REPORT}

Female patient, 41 years old, diagnosed with SLE since 2003 fulfilling ACR criteria: oral ulcers, arthritis, nephritis, ANA, anti-DNA. With lupus nephritis since 2009 (renal biopsy 2010 showing membranous glomerulonephritis) with persistent nephrotic proteinuria refractory to several immunosuppression schemes: high dose corticosteroids, azathioprine, cyclophosphamide $1 \mathrm{~g}$ (6 pulses), cyclosporine. In 2011, mycophenolate mofetil (MMF) was started with good response, but there was a need for suspension in 2016 due to pregnancy. Azathioprine was introduced during this period. After pregnancy, MMF $3 \mathrm{~g} /$ day was restarted without renal response, and multitarget therapy was introduced. The 1 st multitarget regimen was mycophenolate and cyclosporine, the 2 nd regimen mycophenolate and rituximab suspended due to the onset of skin infections, and the 3rd mycophenolate and tacrolimus regimen with no response. Since October 2020, she has been using immunosuppressive therapy with oral cyclophosphamide $100 \mathrm{mg} /$ day associated with moderate doses of corticosteroids in an attempt to control her renal condition. One year ago, she presented cutaneous lesions, with appearance starting in the gluteal region, secretive, with a squamous aspect, hyperemic background, papules some with a purpuric aspect, with a hypothesis of cutaneous vasculitis with secondary bacterial infection. She used several antibiotic regimens without showing better. There was progression with the emergence of new lesions in a disseminated form. A biopsy of a lesion was performed, with culture being confirmed for Fusarium spp. Therapy with liposomal amphotericin B and intravenous voriconazole was instituted and subsequent outpatient treatment with outpatient oral voriconazole with improvement after treatment.

\section{CONCLUSION}

Reports on localized Fusarium infections have mainly described therapeutic and diagnostic difficulties. Cutaneous involvement is common. This description represents a report of this rare opportunistic skin infection in SLE, illustrating the importance of performing skin biopsy to avoid misdiagnosis and treatment delay.

\section{KEYWORDS}

Systemic lupus erythematosus, Fusarium spp., Immunosuppressed, Fungal diseases. 